\section{Review}

Correspondence

Lucinda M. C. Hall

I.m.c.hall@qmul.ac.uk

\title{
Hypermutable bacteria isolated from humans - a critical analysis
}

\author{
Lucinda M. C. Hall and Stephanie K. Henderson-Begg
}

Centre for Infectious Disease, Institute of Cell and Molecular Science, Barts and The London School of Medicine and Dentistry, Queen Mary, University of London, 4 Newark Street, London E1 2AT, UK

\begin{abstract}
Hypermutable bacteria of several species have been described among isolates recovered from humans over the last decade. Interpretation of the literature in this area is complicated by diversity in the determination and definition of hypermutability, and this review outlines the different methods used. Inactivation of the mismatch repair gene mutS is often implicated in the mutator phenotype; the reported effect of mutS inactivation on mutation frequency varies widely between species, from under 10-fold to nearly 1000 -fold, but also varies among different reports on the same species. Particularly high proportions of mutators have been reported among Pseudomonas aeruginosa and other species in the cystic fibrosis lung, epidemic serogroup A Neisseria meningitidis, and Helicobacter pylori. Aspects of the biology of these infections that could be relevant to hypermutability are discussed, and some future directions that may increase our understanding of mutators among bacteria isolated from humans are considered.
\end{abstract}

\section{Introduction}

The survival of species depends on a balance between fidelity of DNA replication and repair, and the generation of variation to allow adaptation to novel environmental challenges. Theoretical analysis argues that, since the majority of mutations that affect phenotype will be deleterious, selection will favour maintenance of the lowest possible mutation rates (Sniegowski et al., 2000). However, under the selection pressure of a novel environment, adaptive mutations would arise at a higher rate in organisms with a mutator phenotype (due for example to inactivation of a mismatch repair gene), and it is possible for mutators to become fixed in a clonal population by 'hitchhiking' with an advantageous mutation elsewhere in the genome. Fixation of mutators has been demonstrated in model systems of Escherichia coli grown in glucose-limiting batch culture or continuous culture, and in the gut of germfree mice (Giraud et al., 2001; Notley-McRobb et al., 2002a, b; Shaver et al., 2002; Sniegowski et al., 1997).

Interest in the topic of hypermutability in bacteria has spread to a wider audience in the last few years, with several reports describing elevated mutation frequencies among clinical isolates of a number of pathogenic species. This review seeks to critically examine recent findings on mutator frequency among bacteria isolated from humans, and to consider how these should be interpreted. In surveying the literature we have become aware of inconsistency in the methods used to study mutation frequency and define mutators, and we therefore devote part of this review to a consideration of these factors.

\section{Determination of mutation frequency}

Rifampicin resistance represents a convenient assay for point mutation that is applicable to diverse bacterial species. Sixty-nine different mutations in $r p o B$ are able to generate rifampicin resistance in E. coli, representing all six possible base substitutions (Garibyan et al., 2003); whether substitutions of all types could also be detected by rifampicin selection in other species is not certain, and is a possible source of differences in mutation frequency between species. It should also be noted that selection for rifampicin resistance cannot detect mutators that predominantly generate frameshift mutations.

We have reviewed 26 published studies that reported the frequency of mutation to rifampicin resistance among a range of bacterial species isolated from humans (Tables 1,2 and 3). The studies aimed either to compare mutation frequencies among collections of clinical isolates, and/or to determine the effect of inactivation of mutS or mutY. Authors made the assumption that the proportion of rifampicin-resistant mutants detected was a measure of the rate of mutation occurring in that culture. In fact the proportion of mutants observed depends not only on mutation rate, but also on the number of generations of growth since each mutation occurred, as demonstrated by the wide distribution of mutant numbers among replicate cultures that was observed in Luria and Delbrück's classic fluctuation test (Luria \& Delbrück, 1943). Although we agree with the consensus view from studies reviewed here that determination of mutation rate is unnecessary in this context, we suggest some guidelines that should permit 
Table 1. Prevalence of hypermutators among clinical isolates

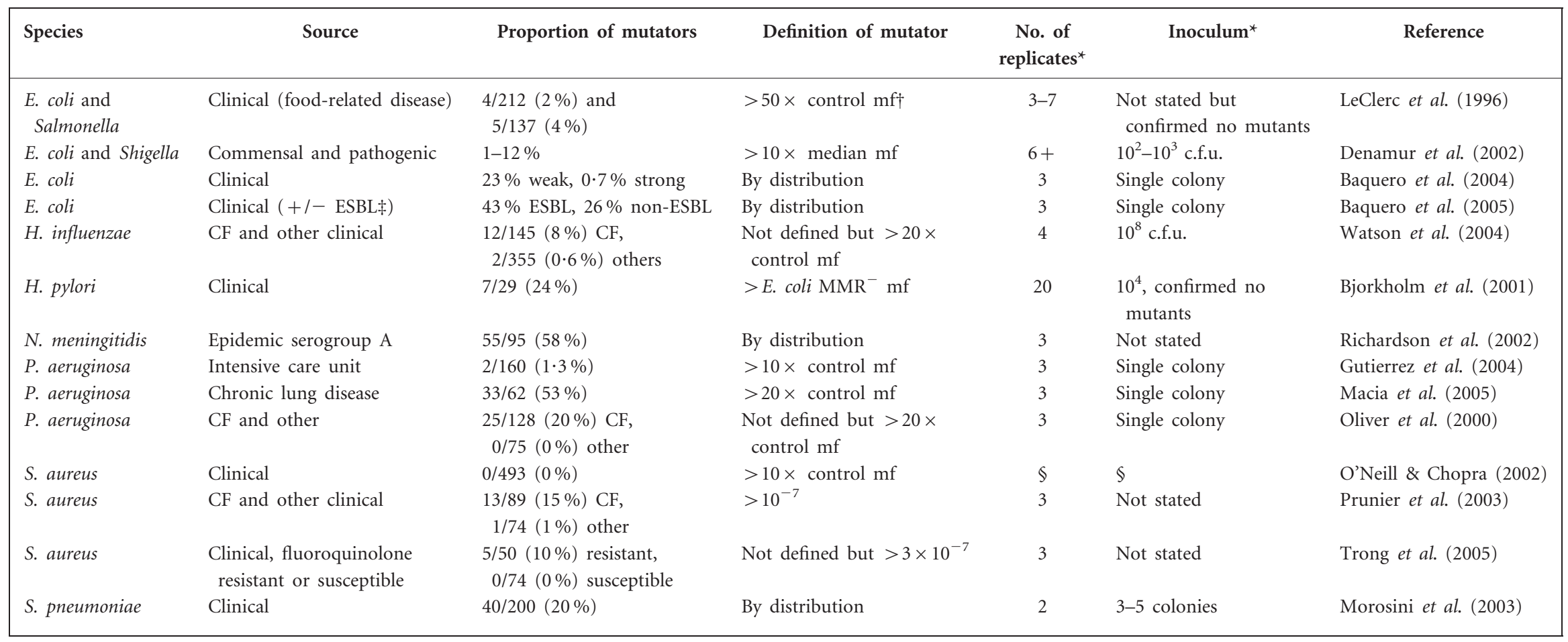

${ }^{\star}$ In some studies rapid preliminary screening was conducted by simpler methodology.

$\dagger \mathrm{mf}$, mutation frequency to rifampicin resistance.

‡ESBL, extended-spectrum $\beta$-lactamase.

$\S$ Rapid preliminary screening (more likely to give false positives than false negatives); personal communication, A. O’Neill, University of Leeds, UK. 
Table 2. Effect of inactivation of mutS on mutation frequency to rifampicin resistance

\begin{tabular}{|c|c|c|c|c|c|c|}
\hline \multirow[t]{2}{*}{ Species } & \multicolumn{2}{|c|}{ Mutation frequency } & \multirow{2}{*}{$\begin{array}{c}\text { mutS } \\
\text { increase }\end{array}$} & \multirow{2}{*}{$\begin{array}{c}\text { No. of } \\
\text { replicates }\end{array}$} & \multirow[t]{2}{*}{ Inoculum } & \multirow[t]{2}{*}{ Reference } \\
\hline & Wild-type ${ }^{\star}$ & $m u t S^{*}$ & & & & \\
\hline E. coli & $3 \cdot 2 \times 10^{-9}$ & $3 \cdot 0 \times 10^{-6}$ & 950 & $5-8$ & $<100$ cells & Pang et al. (1985) \\
\hline E. coli & $4.9 \times 10^{-8}$ & $1 \cdot 0 \times 10^{-6}$ & 20 & 3 & $<10^{4}$ cells $\dagger$ & Oliver et al. (2002a) \\
\hline E. coli & $4 \cdot 0 \times 10^{-8}$ & $2 \cdot 2 \times 10^{-6}$ & 55 & 3 & Single colony & Miller et al. (2002) \\
\hline H. influenzae & $1 \cdot 2 \times 10^{-9}$ & $5 \cdot 0 \times 10^{-8}$ & 42 & 4 & $10^{8}$ cells & Watson et al. (2004) \\
\hline N. meningitidis & $1-7 \times 10^{-9}$ & $1-4 \times 10^{-8}$ & $1-20$ & $10+$ & Not stated & Richardson \& Stojiljkovic (2001) \\
\hline N. meningitidis & $2 \cdot 7 \times 10^{-9}$ & $3 \cdot 0 \times 10^{-8}$ & 11 & $8+$ & Single colony plate & Martin et al. (2004) \\
\hline P. aeruginosa & $1 \cdot 2 \times 10^{-8}$ & $5 \cdot 8 \times 10^{-6}$ & 480 & 3 & Single colony & Gutierrez et al. (2004) \\
\hline S. typhimurium & $4 \cdot 4 \times 10^{-9}$ & $2 \cdot 2 \times 10^{-6}$ & 500 & $5-8$ & $<100$ cells & Pang et al. (1985) \\
\hline S. pneumoniae & $2 \cdot 6 \times 10^{-8}$ & $1 \cdot 1 \times 10^{-7}$ & 4 & 2 & 3-5 colonies & Morosini et al. (2003) \\
\hline
\end{tabular}

${ }^{*}$ Where more than one figure is given the values are for different isolates except where noted.

$\dagger$ Personal communication, J. Blazquez, Centro Nacional de Biotecnología, Madrid, Spain.

$\ddagger$ Personal communication, A. O’Neill, University of Leeds, UK.

$\S$ Values for same organism carrying cloning vector.

comparable measures of mutation frequency in diverse organisms (see Fig. 1). The accurate determination of mutation rate has been described and discussed elsewhere (Crane et al., 1996; Drake, 1991; Rosche \& Foster, 2000).

In two studies reviewed, the authors failed both to specify the inoculum used and to specify the number of replicate cultures used to determine mutation frequency, giving the impression that the figures derived from a single culture. On this basis it is impossible to judge the validity of the results presented and these reports were discounted from further analysis. Seven further studies did not specify the inoculum used (nor state that pre-existing mutants were excluded), although in each case at least three replicate cultures were performed. Corresponding authors were emailed for clarification, and additional details provided are recorded in Tables 1 and 2.

\section{Definition of mutators and hypermutators}

Historically, genes defined by the phenotype of elevated mutation rate have been named mut for mutator, and strains bearing mutator alleles are known as mutator strains (or simply as mutators). In recent years, and particularly in studies of clinical isolates, it has become common to refer to strains with elevated mutation frequency as hypermutators, in line with their phenotype of hypermutability. No distinction has been made in the literature between mutators and hypermutators, and we will consider the two terms to be interchangeable. While it is relatively

Table 3. Effect of inactivation of mutY on mutation frequency to rifampicin resistance

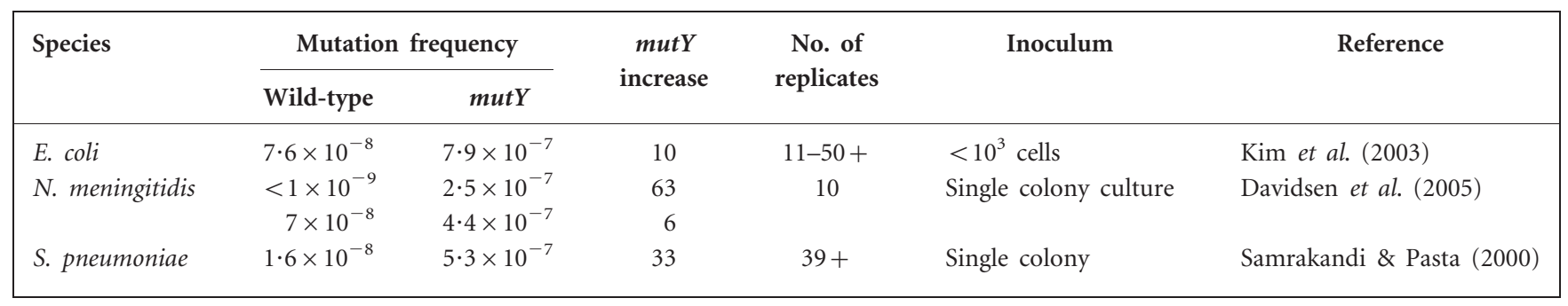


- Inoculate three or more replicate broths with a standardized inoculum*

- Use more replicates where small differences in frequency are to be compared or where quantification is inaccurate (e.g. due to low number of mutant colonies)

- Grow cultures to maximum viable cell count by a standardized protocol

- For organisms that lose viability in stationary phase monitoring of growth is critical

- Spread aliquots on selective plates and (with dilution) on non-selective plates

- Calculate median mutation frequency (total no. of mutants/total viable count)

Use of the median avoids bias due to possible 'jack-pot' cultures where mutation occurred at an early stage in growth

- Conduct statistical analyses (for example to compare mutation frequency between strains) using tests designed for non-parametric data (e.g. Mann-Witney U-test)

- Avoid use of standard deviation for the same reason

*The standardized inoculum

- Option A: One or a few colonies from a fresh plate

- Quick and simple

- Viable count unknown and possibility of mutants in inoculum not excluded

- But ensures all mutants occurred within standard time frame (since colony founder)

- Option B: Known number of c.f.u. (usually $10^{2}-10^{4}$ ) from characterized starter culture

- Probability of pre-existing mutants can be quantified and minimized

- If pre-existing mutants cannot be excluded from inoculum (because of high mutation frequency and/or requirement for high viable count), the starter culture should be prepared with a minimum or fixed number of steps from a single colony to ensure comparability
Fig. 1. Guidelines for routine determination of mutation frequency. straightforward to define variants of a laboratory strain as having an elevated mutation frequency, this becomes much more challenging when investigating populations of bacteria isolated from 'the wild', in this context from clinical specimens. Among the studies of bacteria isolated from humans reviewed here, two alternative strategies have been used to provide a working definition of a mutator (Table 1).

The first strategy to define mutators involves identifying isolates that lie outside the 'normal' distribution of mutation frequencies for a collection of independent isolates of the same species. The strategy is only possible where relatively large numbers of isolates are being investigated, since it is apparent from many of the studies reviewed here that a wide range of mutation frequencies is found even among 'normal' isolates. For example Richardson et al. (2002) examined 95 isolates of epidemic serogroup A Neisseria meningitidis and demonstrated that mutation frequencies were clustered into two distinct classes with means of $2.6 \times 10^{-9}$ and $2.3 \times 10^{-8}$ rifampicin-resistant mutants per c.f.u. Although the class with higher mutation frequency actually represented the majority of the population $(57 \%)$, at least some of these isolates could have a 'normal' or lower mutation frequency restored by complementation with functional mutS or $m u t L$, suggesting that they do represent a hypermutable set of organisms. A study of hypermutable Pseudomonas aeruginosa in cystic fibrosis patients by Oliver et al. (2000) does not specifically address the definition of mutators, but their published data do show a distinction between the normal population, mean frequency of rifampicin-resistant mutants $2 \cdot 9 \times 10^{-8}$, and mutators, mean mutation frequency $3 \cdot 6 \times 10^{-6}$. Again the ability of mutS to complement the hypermutable phenotype in at least some isolates provides additional support to the biological significance of the definition used.

Other authors who have sought to separate hypermutators from the normal population have been caught out by a mathematical error. Specifically, Morosini et al. (2003) and Baquero et al. (2004) both plotted the distribution of mutation frequencies in populations by grouping isolates into frequency intervals. However, these intervals were neither arithmetically nor geometrically equal: if values are converted to mutants per $10^{8}$ c.f.u., isolates were grouped into intervals of $5-7 \cdot 5,7 \cdot 5-10,10-25,25-50$, etc. In this situation frequencies that are evenly distributed along a logarithmic scale would tend to appear clustered in the intervals that represent the widest geometric range of values (e.g. 10-25, which represents a 2.5-fold range), and sparse in the intervals that represent smaller ranges (e.g. 7·5-10, representing a 1.3-fold range). It is not possible to determine from the data presented in these studies whether the putative mutators genuinely form a distinct population. In the case of Morosini et al. (2003), the absence of hexA and hexB mutations (equivalent to muts and mutL in other species) among putative mutators of Streptococcus 
pneumoniae may indicate that these isolates are not really distinct from the general population (but see below for discussion of the impact of hex mutants in S. pneumoniae).

The alternative strategy to defining mutators is simply to set a value for frequency of mutants above which an isolate will be considered to be a mutator. This may either be at a certain fold higher than a control strain, typically $10 \times$ or $50 \times$, or an absolute value such as $>10^{-7}$. In many of the studies applying this strategy a biological justification for the cut-off value is presented, such as the mutation frequency of a mutS inactivation mutant (Table 2). It becomes evident, as discussed below, that the effect of inactivation of mismatch repair genes differs considerably among species and thus affects the definitions used. On this basis it does not seem fruitful to recommend a single working definition of a mutator, since this would be unlikely to be applicable to all studies. However, the reader should be aware that the literature encompasses many different definitions, and that in comparing studies on different species we may not be comparing like with like. We draw attention to anomalies that we consider may be significant in the sections that follow.

\section{The effects of muts and mutY inactivation on mutation frequency in diverse species}

We have reviewed the impact of mutS inactivation in a variety of human pathogens where the effect on mutation frequency to rifampicin resistance has been reported. A summary of the results reported is presented in Table 2 . Strikingly, the estimated increase in mutation frequency between wild-type organisms and their isogenic mutS mutants varies from fourfold in S. pneumoniae to 950fold in E. coli. However, these estimates should be viewed in the context of the considerations noted above, and the highly divergent results reported in some cases within species. For example, the reported increase in mutation frequency conferred by inactivation of $m u t S$ in $E$. coli ranges from 20 -fold to 950 -fold. The two most divergent results arise from studies of inactivation of mutS in the same laboratory isolate, AB1157: while mutation frequencies reported for the mutS mutants were similar in both studies $\left(1-3 \times 10^{-6}\right)$, the mutation frequency for the parent isolate was estimated at $3 \cdot 2 \times 10^{-9}$ by one group and $4.9 \times 10^{-8}$ by the other (Oliver et al., 2002a; Pang et al., 1985). Thus inaccuracy in determination of mutation frequency (particularly at low frequency, where the number of colonies detected is usually small) is strongly implicated in the differences reported.

From the data reported in Table 2 it appears that, in E. coli, Salmonella typhimurium, $P$. aeruginosa and Staphylococcus aureus, inactivation of muts increases the mutation frequency to rifampicin resistance by around two orders of magnitude, to at least $1 \times 10^{-6}$. Haemophilus influenzae, $N$. meningitidis and S. pneumoniae appear to fall into a different category, with mutS inactivation increasing mutation by around one order of magnitude, resulting in mutation frequencies of no more than $1 \times 10^{-7}$. Acinetobacter would fall into an intermediate category by criteria of both fold-increase and absolute mutation frequency. These results indicate a tendency for the naturally transformable species to have relatively low increases in mutation frequency upon inactivation of mutS, while those characterized as largely clonal in population structure are much more strongly affected. This may reflect the stronger barrier to interspecies recombination formed by the mismatch repair systems of the clonal species, versus relatively permissive recombination in transformable species such as $S$. pneumoniae. In the transformable species it must be assumed that other systems are more important than mismatch repair in maintaining low mutation rates.

Reports have been published on the effect of inactivation of $m u t Y$ on frequency of mutation to rifampicin resistance for three of the species in which mutS has also been investigated (Table 3). Interestingly, mut $Y$ mutants of $N$. meningitidis and S. pneumoniae had mutation frequencies as high as or higher than mutS mutants, while in E. coli the mutY mutation frequency was an order of magnitude lower than that for $m u t S$ (and similar to the frequency seen for $m u t Y$ in the other species) (Davidsen et al., 2005; Kim et al., 2003; Samrakandi \& Pasta, 2000). The DNA glycosylase activity of MutY has a critical role in the protection of DNA against oxidative damage and may be particularly relevant to bacterial pathogens contending with oxygen radicals released by the immune system. It should also be noted that overexpression of $m u t S$ in E. colican compensate for the loss of mut $Y$ activity (Zhao \& Winkler, 2000), meaning that caution is required in the interpretation of complementation tests to determine the genes involved in mutator phenotypes.

\section{How common are mutators among bacteria isolated from humans?}

We have searched the literature for reports on the isolation of hypermutable bacteria from clinical sources, and summarize the results observed in Table 1 . In the absence of a selective advantage it would be predicted that mutators, arising as mutants of a small number of loci, would occur by chance at a prevalence of no more than $1 \times 10^{-5}$, at least in E. coli (Mao et al., 1997).

Several studies since the mid-1990s have investigated the frequency of mutators among E. coli isolated from humans, including pathogenic and commensal isolates (Baquero et al., 2004, 2005; Denamur et al., 2002; LeClerc et al., 1996). Estimates varied both by study and by source of isolate (see also below), and different criteria were used to define mutators. LeClerc et al. (1996) defined putative mutators as those with $>50 \times$ higher frequency of rifampicin-resistant mutants than controls (effectively $>5 \times 10^{-7}$ ), but discounted many by further testing for an elevated frequency of mutants resistant to other agents. Confirmed mutators all had mutation frequencies to rifampicin resistance of $>1 \times 10^{-6}$, and represented $2 \%$ of $E$. coli investigated, 
much higher than would be expected by chance. (A similar prevalence, $4 \%$, was observed among Salmonella isolates.) Seven of nine mutators were suggested to have mutS defects by complementation, and in four of four isolates investigated further this was confirmed by the presence of large deletions ( $\mathrm{Li}$ et al., 2003). The even higher prevalence of mutators reported in some other studies had less stringent criteria, with isolates being considered mutators if they had mutation frequencies to rifampicin resistance greater than 4 or $5 \times 10^{-8}$ (Baquero et al., 2004, 2005; Denamur et al., 2002). However, $7 \cdot 5 \%$ of $E$. coli from urinary tract infection were classified by Denamur et al. (2002) as 'high mutators' with mutation frequencies $>2.5 \times 10^{-7}$ (and confirmed with other antimicrobials), and this was significantly greater than the proportion of 'high mutators' in isolates from other sources.

Particular attention was brought to the issue of hypermutability in 2000 by the report that $20 \%$ of 128 isolates of $P$. aeruginosa from the lungs of patients with cystic fibrosis (CF) were mutators, while none were found among $75 P$. aeruginosa from other sources (Oliver et al., 2000). All mutators had mutation frequencies that were over $1 \times 10^{-7}$, and, as discussed above, formed a separate distribution peak from other isolates. Four of 11 mutator isolates investigated were considered to have mutS defects on the basis of complementation. It has since been reported that mutators are also common among $P$. aeruginosa from patients with other chronic lung conditions, but not among isolates collected from patients in intensive care (Macia et al., 2005).

Isolates of other species from patients with CF have also been reported to include high proportions of mutators. Eight per cent of $H$. influenzae from CF (versus $0.6 \%$ from other infections) were classified as mutators and had reported mutation frequencies to rifampicin resistance ranging from $2 \times 10^{-8}$ to $5 \times 10^{-6}$ [the authors acknowledge that due to the high inoculum required for broth cultures of this organism pre-existing mutants could not be excluded for isolates with high mutation rates, but the same isolates also gave high mutant frequencies for nalidixic acid resistance (Watson et al., 2004)]. Hypermutability was associated with polymorphism at conserved sites of mutS in seven of eight isolates. Mutators defined by mutation frequency to rifampicin resistance $>1 \times 10^{-7}$ represented $15 \%$ of 89 S. aureus isolates from CF, and only $1 \%$ of isolates from blood or respiratory tract (Prunier et al., 2003). This threshold for mutators appears low in relation to control laboratory strains, which have been reported by the same authors and others to have mutation frequencies close to or slightly over the same value (O'Neill \& Chopra, 2002; Prunier \& Leclercq, 2005), although most clinical isolates did have lower mutation frequencies. From the graphs presented, 4 of $89 \mathrm{CF}$ and 2 of 74 non-CF isolates had particularly high mutation frequencies (close to $1 \times 10^{-6}$ or above) (Prunier et al., 2003). Deletions or polymorphisms were detected in mutS or mutL in some of the reported mutators, but the mutation frequencies of these particular isolates was not specified (Prunier \& Leclercq, 2005). High frequencies of mutators have also been reported among $S$. pneumoniae from CF patients (del Campo et al., 2005). We have discussed earlier some doubts about the definition of mutators in this species, but, as with $S$. aureus, the data presented show a clear tendency for a greater proportion of $\mathrm{CF}$ isolates to have higher mutation frequencies than those of isolates from other sources, and a sizeable proportion were more mutable than a laboratory hexA (mutS) mutant (del Campo et al., 2005; Morosini et al., 2003).

A strikingly high frequency of hypermutability has been reported among epidemic serogroup A $N$. meningitidis, with $57 \%$ of isolates considered to have an elevated mutation frequency to rifampicin resistance (Richardson et al., 2002). Eleven of 54 hypermutators were deduced to have defects in mutS or mutL by complementation.

A study of 29 clinical isolates of Helicobacter pylori demonstrated a 700 -fold range of mutation frequency (Bjorkholm et al., 2001). It was considered that $25 \%$ of these isolates could be classified as hypermutators since their frequencies of mutation to rifampicin resistance exceeded that of E. coli mismatch repair mutants, at $>1 \cdot 2 \times 10^{-6}$; the genetic basis of increased mutability was not determined, but this species lacks a homologue of E. coli mutS and inactivation of the related gene mutS2 did not increase mutation frequency.

\section{How should mutators among clinical isolates be interpreted?}

There can be no doubt that organisms with elevated rates of mutation are isolated from clinical sources more commonly than would be expected by chance (i.e. if they existed at the frequency expected in a population grown from a single cell), despite concerns regarding methodology and interpretation in some published reports. Furthermore it appears that mutators are found more commonly among isolates from some types of clinical specimen than others. It should also be recognized that even among organisms without an overt mutator phenotype, a wide range of mutation frequencies is detected (typically a 10- to 100-fold range among the studies described in Table 1), and in some studies the distribution of isolates within this range differs among isolates from different sources (see above).

One of the principal motivations for research on mutators in bacteria isolated from humans has been the risk posed for selection of antibiotic resistance, as discussed in depth by others (Blazquez, 2003; Chopra et al., 2003). Laboratory studies have illustrated that mutators in experimental populations of E. coli can be amplified to high frequency by few rounds of selection (for example by antibiotics), and furthermore that mutagenesis followed by selection increases the likelihood that mutators will proliferate (Mao et al., 1997). In a number of the studies included in Table 1, the prevalence of mutators was reported to be higher among antibiotic-resistant than susceptible isolates 
(Baquero et al., 2005; Macia et al., 2005; Prunier et al., 2003), although this was not the case in $H$. pylori or S. pneumoniae (Bjorkholm et al., 2001; Morosini et al., 2003). As well as providing a selection pressure, antibiotics may also have a role in mutagenesis through promotion of transient increases in mutation frequency (Blazquez et al., 2002; Gillespie et al., 2005; Henderson-Begg et al., 2006; PerezCapilla et al., 2005). More attention should perhaps also be given to the mutagenic effects of reactive oxygen species released by the immune system to combat infection. This may be particularly relevant in chronic infections such as the CF lung (Ciofu et al., 2005).

A question that has not to our knowledge been addressed thoroughly is how mutators are distributed among patients. If, for example, $7 \cdot 5 \%$ of $E$. coli isolates in a collection cultured from urinary tract infections are mutators, does this mean that within each patient around $7 \cdot 5 \%$ of infecting bacteria are mutators, or that $7 \cdot 5 \%$ of patients are infected with a population of bacteria of which the majority are mutators (while mutators are very rare in the remainder of patients)? The collections of clinical isolates readily available to research groups usually represent single 'typical' colonies each selected from a different patient specimen. Unless a mutator carries a distinct phenotype, the probability of a mutator colony being picked should match the proportion of mutators in the infecting population of an individual patient, so the two situations described above would yield the same result. Information from this type of collection cannot enable us to distinguish between these two extremes, yet it may be very pertinent to understanding the risk of selecting for antibiotic resistance during treatment. $P$. aeruginosa mutators were isolated in specimens collected over a number of years from the same CF patients, suggesting that, at least in this context, mutators do predominate and persist in some patients (Oliver et al., 2000).

How does the prevalence of mutators relate to the biology of bacterial pathogens? $P$. aeruginosa is an opportunistic pathogen with a large genome that allows ready adaptation to different environments. Selection of mutations adaptive to growth in the lung, selection of resistance mutants by multiple rounds of antimicrobial chemotherapy, and mutagenic effects of chronic inflammation may all contribute to the proliferation of mutators in the population infecting an individual patient. Counterselection against some of the potentially deleterious effects of a mutator phenotype may be weak since, for example, auxotrophic mutants can readily be recovered from the CF lung (Thomas et al., 2000). The long-term significance of mutators in the CF lung (beyond their effect on an individual patient) depends on whether the highly adapted strains that evolve can be transmitted to other patients, and whether they then result in more serious or rapidly progressing infection. Since there is evidence that cross-infection between CF patients can occur, this is clearly an issue of concern.

A different situation applies with $N$. meningitidis, which lives primarily in the human nasopharynx and, at frequencies that depend on both host and pathogen factors, invades the bloodstream and/or central nervous system. Transmission occurs principally via carriage, so that invasion may be considered an evolutionary dead-end. Expression of many surface markers involved in interaction with the host is subject to phase variation mediated by 'contingency genes' that switch at high frequency via changes of repeat number within regions of simple repetitive DNA. A strong correlation was observed between general mutation rate and the rate of phase variation, so the very high frequency of mutators found in epidemic serogroup A isolates may be related to selection for increased phase variation (Richardson et al., 2002). To understand more about the biological significance of mutators in this species it would be important to know whether these occur as often in carriage isolates as in invasive isolates. In an earlier study of serogroup B N. meningitidis, elevated mutation frequency also appeared to correlate with an increased rate of capsule variation, although this was only measured in six isolates (Bucci et al., 1999). Both phenotypes were attributed to the absence of DNA methyltransferase (Dam) activity (required to identify foreign DNA during mismatch repair), as confirmed by insertional inactivation of dam. Intriguingly, Bucci et al. (1999) found that 22/22 serogroup B isolates from invasive disease lacked Dam activity and were hence predicted to be hypermutators, while Dam was active in 11/21 serogroup B isolates from the nasopharynx. However, Richardson \& Stojiljkovic (2001) reported that Dam status did not correlate with mutation frequency among serogroup A isolates in their study.

$H$. pylori is also an obligate parasite of humans that causes disease in only a portion of affected individuals, but little is known about the factors involved in moving between asymptomatic carriage and destructive infection. The genome of this species is exceptionally plastic, which may relate to the absence of a mismatch repair system homologous to that of E. coli and most other bacterial pathogens. It is difficult to assess how mutators could usefully be defined in $\mathrm{H}$. pylori, but it is evident that some strains mutate at much higher frequencies than others (Bjorkholm et al., 2001). Isolates were obtained from patients with non-ulcer dyspepsia as well as peptic ulcer and gastric cancer patients, and there was no significant difference in the mutation frequencies obtained, although perhaps a trend for lower frequencies among the non-ulcer dyspepsia patients. It would be of interest to know whether mutation frequencies were as high in asymptomatic carriage isolates. However, the need for endoscopy to recover organisms from the stomach is likely to make this impracticable. Even the least mutable isolates described by Bjorkholm et al. (2001) have mutation frequencies that would be considered quite high in other species; it might be useful to consider the frequency of mutators that 'would be expected by chance' in this species by testing in populations grown from single colonies.

\section{Concluding remarks}

The past decade has seen a surge of interest in the mutation 
rates of bacteria isolated from humans. Sophisticated genetic systems developed in E. coli for detection of particular types of mutation are not readily applicable to clinical isolates of diverse species, and most of the studies that we have reviewed have relied on mutation to rifampicin resistance as a measure of general mutation frequency. In many cases hypermutability to rifampicin resistance was supported by demonstrating parallel hypermutability to other agents. Nevertheless we would urge a greater clarity in publication of the methods used to determine mutation frequency, particularly in those species that may not be amenable to the standard methods used in E. coli.

Investigation of the mechanisms responsible for elevated mutation frequencies has largely focused on mutS homologues and other genes of the (methyl-directed) mismatch repair system. Mutations in these genes are strongly implicated in some mutators by virtue of sequencing and/ or complementation, although their importance may have been overestimated where complementation alone was relied upon due to potential compensation by mutS of mutY defects (Zhao \& Winkler, 2000). In any case a significant proportion of mutators could not be accounted for by mutS deficiency. Other candidates as mutator genes among clinical isolates would clearly be mut $Y$ and mut $T$, which are involved in protection from and repair of oxidative damage such as the effects of oxygen radicals released by cells of the immune system; homologues of these genes are reported on the COG database in most taxonomic groups that include human pathogens (Tatusov et al., 2003). However, we note that cloned $m u t T$ (and also mutM) failed to complement any of 11 mutator strains of $P$. aeruginosa that had been isolated from CF patients (Oliver et al., 2002b).

The clinical significance of mutators is still subject to debate. In a situation where a high proportion of the bacterial population infecting an individual is hypermutable, the probability of selecting resistant mutants during antibiotic treatment must clearly be increased. This would be pertinent to the treatment of any chronic or long-term infections, including those associated with CF. Similarly mutators would increase the probability of selecting strains more highly adapted to conditions within the infected host (although not necessarily more effective in transmission to a new host). Whether mutators have made a major contribution to the generation of antibiotic resistance (or increased pathogenicity) in a wider context (outside chronic infection) is much more difficult to judge: as mentioned earlier, evidence of association between resistance and hypermutability for different species is conflicting; on the other hand, reversion of the hypermutator phenotype subsequent to the acquisition of resistance could obscure a role of hypermutability among antibiotic-resistant bacteria isolated from patients.

We have limited this review to an analysis of stable or constitutive mutators. However, the reader should be aware of parallel studies on transient hypermutability induced by stress. It is of particular interest to note that, in a large collection of E. coli, an inverse relationship has been demonstrated between constitutive mutation frequency and the induction of increased mutation frequency in ageing colonies (Bjedov et al., 2003). A number of reviews discussing stress-induced mutagenesis, both in general and in relation to antibiotic resistance, have been published recently by others (Blazquez, 2003; Bridges, 2001; Chopra et al., 2003; Foster, 2005; Rosenberg et al., 1998).

\section{Acknowledgements}

We thank the Department of Health and St Bartholomew's and The Royal London Charitable Foundation for research support.

\section{References}

Baquero, M. R., Nilsson, A. I., Turrientes Mdel, C., Sandvang, D., Galan, J. C., Martinez, J. L., Frimodt-Moller, N., Baquero, F. \& Andersson, D. I. (2004). Polymorphic mutation frequencies in Escherichia coli: emergence of weak mutators in clinical isolates. J Bacteriol 186, 5538-5542.

Baquero, M. R., Galan, J. C., del Carmen Turrientes, M., Canton, R., Coque, T. M., Martinez, J. L. \& Baquero, F. (2005). Increased mutation frequencies in Escherichia coli isolates harboring extended-spectrum beta-lactamases. Antimicrob Agents Chemother 49, 4754-4756.

Bjedov, I., Tenaillon, O., Gerard, B., Souza, V., Denamur, E., Radman, M., Taddei, F. \& Matic, I. (2003). Stress-induced mutagenesis in bacteria. Science 300, 1404-1409.

Bjorkholm, B., Sjolund, M., Falk, P. G., Berg, O. G., Engstrand, L. \& Andersson, D. I. (2001). Mutation frequency and biological cost of antibiotic resistance in Helicobacter pylori. Proc Natl Acad Sci U S A 98, 14607-14612.

Blazquez, J. (2003). Hypermutation as a factor contributing to the acquisition of antimicrobial resistance. Clin Infect Dis 37, 1201-1209.

Blazquez, J., Oliver, A. \& Gomez-Gomez, J. M. (2002). Mutation and evolution of antibiotic resistance: antibiotics as promoters of antibiotic resistance? Curr Drug Targets 3, 345-349.

Bridges, B. A. (2001). Hypermutation in bacteria and other cellular systems. Philos Trans R Soc Lond B Biol Sci 356, 29-39.

Bucci, C., Lavitola, A., Salvatore, P., Del Giudice, L., Massardo, D. R., Bruni, C. B. \& Alifano, P. (1999). Hypermutation in pathogenic bacteria: frequent phase variation in meningococci is a phenotypic trait of a specialized mutator biotype. Mol Cell 3, 435-445.

Chopra, I., O'Neill, A. J. \& Miller, K. (2003). The role of mutators in the emergence of antibiotic-resistant bacteria. Drug Resist Updat 6, 137-145.

Ciofu, O., Riis, B., Pressler, T., Poulsen, H. E. \& Hoiby, N. (2005). Occurrence of hypermutable Pseudomonas aeruginosa in cystic fibrosis patients is associated with the oxidative stress caused by chronic lung inflammation. Antimicrob Agents Chemother 49, 2276-2282.

Crane, G. J., Thomas, S. M. \& Jones, M. E. (1996). A modified LuriaDelbruck fluctuation assay for estimating and comparing mutation rates. Mutat Res 354, 171-182.

Davidsen, T., Bjoras, M., Seeberg, E. C. \& Tonjum, T. (2005). Antimutator role of DNA glycosylase MutY in pathogenic Neisseria species. J Bacteriol 187, 2801-2809. 
del Campo, R., Morosini, M. I., de la Pedrosa, E. G., Fenoll, A., Munoz-Almagro, C., Maiz, L., Baquero, F. \& Canton, R. (2005). Population structure, antimicrobial resistance, and mutation frequencies of Streptococcus pneumoniae isolates from cystic fibrosis patients. J Clin Microbiol 43, 2207-2214.

Denamur, E., Bonacorsi, S., Giraud, A. \& 8 other authors (2002). High frequency of mutator strains among human uropathogenic Escherichia coli isolates. J Bacteriol 184, 605-609.

Drake, J. W. (1991). A constant rate of spontaneous mutation in DNA-based microbes. Proc Natl Acad Sci U S A 88, 7160-7164.

Foster, P. L. (2005). Stress responses and genetic variation in bacteria. Mutat Res 569, 3-11.

Garibyan, L., Huang, T., Kim, M. \& 8 other authors (2003). Use of the $r p o B$ gene to determine the specificity of base substitution mutations on the Escherichia coli chromosome. DNA Repair 2, 593-608.

Gillespie, S. H., Basu, S., Dickens, A. L., O'Sullivan, D. M. \& McHugh, T. D. (2005). Effect of subinhibitory concentrations of ciprofloxacin on Mycobacterium fortuitum mutation rates. J Antimicrob Chemother 56, 344-348.

Giraud, A., Matic, I., Tenaillon, O., Clara, A., Radman, M., Fons, M. \& Taddei, F. (2001). Costs and benefits of high mutation rates: adaptive evolution of bacteria in the mouse gut. Science 291, 2606-2608.

Gutierrez, O., Juan, C., Perez, J. L. \& Oliver, A. (2004). Lack of association between hypermutation and antibiotic resistance development in Pseudomonas aeruginosa isolates from intensive care unit patients. Antimicrob Agents Chemother 48, 3573-3575.

Henderson-Begg, S. K., Livermore, D. M. \& Hall, L. M. (2006). Effect of subinhibitory concentrations of antibiotics on mutation frequency in Streptococcus pneumoniae. J Antimicrob Chemother 57, 849-854.

Kim, M., Huang, T. \& Miller, J. H. (2003). Competition between MutY and mismatch repair at $\mathrm{A} \times \mathrm{C}$ mispairs in vivo. J Bacteriol 185, 4626-4629.

LeClerc, J. E., Li, B., Payne, W. L. \& Cebula, T. A. (1996). High mutation frequencies among Escherichia coli and Salmonella pathogens. Science 274, 1208-1211.

Li, B., Tsui, H. C., LeClerc, J. E., Dey, M., Winkler, M. E. \& Cebula, T. A. (2003). Molecular analysis of mutS expression and mutation in natural isolates of pathogenic Escherichia coli. Microbiology 149, 1323-1331.

Luria, S. E. \& Delbrück, M. (1943). Mutations of bacteria from virus sensitivity to virus resistance. Genetics 28, 491-511.

Macia, M. D., Blanquer, D., Togores, B., Sauleda, J., Perez, J. L. \& Oliver, A. (2005). Hypermutation is a key factor in development of multiple-antimicrobial resistance in Pseudomonas aeruginosa strains causing chronic lung infections. Antimicrob Agents Chemother 49, 3382-3386.

Mao, E. F., Lane, L., Lee, J. \& Miller, J. H. (1997). Proliferation of mutators in a cell population. J Bacteriol 179, 417-422.

Martin, P., Sun, L., Hood, D. W. \& Moxon, E. R. (2004). Involvement of genes of genome maintenance in the regulation of phase variation frequencies in Neisseria meningitidis. Microbiology 150, 3001-3012.

Miller, K., O’Neill, A. J. \& Chopra, I. (2002). Response of Escherichia coli hypermutators to selection pressure with antimicrobial agents from different classes. J Antimicrob Chemother 49, 925-934.

Morosini, M. I., Baquero, M. R., Sanchez-Romero, J. M., Negri, M. C., Galan, J. C., del Campo, R., Perez-Diaz, J. C. \& Baquero, F. (2003). Frequency of mutation to rifampin resistance in Streptococcus pneumoniae clinical strains: hexA and hexB polymorphisms do not account for hypermutation. Antimicrob Agents Chemother 47, 1464-1467.
Notley-McRobb, L., Pinto, R., Seeto, S. \& Ferenci, T. (2002a). Regulation of mutY and nature of mutator mutations in Escherichia coli populations under nutrient limitation. J Bacteriol 184, 739-745.

Notley-McRobb, L., Seeto, S. \& Ferenci, T. (2002b). Enrichment and elimination of mutY mutators in Escherichia coli populations. Genetics 162, 1055-1062.

Oliver, A., Canton, R., Campo, P., Baquero, F. \& Blazquez, J. (2000). High frequency of hypermutable Pseudomonas aeruginosa in cystic fibrosis lung infection. Science 288, 1251-1254.

Oliver, A., Baquero, F. \& Blazquez, J. (2002a). The mismatch repair system (mutS, mutL and uvrD genes) in Pseudomonas aeruginosa: molecular characterization of naturally occurring mutants. Mol Microbiol 43, 1641-1650.

Oliver, A., Sanchez, J. M. \& Blazquez, J. (2002b). Characterization of the GO system of Pseudomonas aeruginosa. FEMS Microbiol Lett 217, 31-35.

O'Neill, A. J. \& Chopra, I. (2002). Insertional inactivation of mutS in Staphylococcus aureus reveals potential for elevated mutation frequencies, although the prevalence of mutators in clinical isolates is low. J Antimicrob Chemother 50, 161-169.

Pang, P. P., Lundberg, A. S. \& Walker, G. C. (1985). Identification and characterization of the mutL and mutS gene products of Salmonella typhimurium LT2. J Bacteriol 163, 1007-1015.

Perez-Capilla, T., Baquero, M. R., Gomez-Gomez, J. M., Ionel, A., Martin, S. \& Blazquez, J. (2005). SOS-independent induction of $\operatorname{din} B$ transcription by beta-lactam-mediated inhibition of cell wall synthesis in Escherichia coli. J Bacteriol 187, 1515-1518.

Prunier, A. L. \& Leclercq, R. (2005). Role of mutS and mutL genes in hypermutability and recombination in Staphylococcus aureus. J Bacteriol 187, 3455-3464.

Prunier, A. L., Malbruny, B., Laurans, M., Brouard, J., Duhamel, J. F. \& Leclercq, R. (2003). High rate of macrolide resistance in Staphylococcus aureus strains from patients with cystic fibrosis reveals high proportions of hypermutable strains. J Infect Dis 187, 1709-1716.

Richardson, A. R. \& Stojiljkovic, I. (2001). Mismatch repair and the regulation of phase variation in Neisseria meningitidis. Mol Microbiol 40, 645-655.

Richardson, A. R., Yu, Z., Popovic, T. \& Stojiljkovic, I. (2002). Mutator clones of Neisseria meningitidis in epidemic serogroup A disease. Proc Natl Acad Sci U S A 99, 6103-6107.

Rosche, W. A. \& Foster, P. L. (2000). Determining mutation rates in bacterial populations. Methods 20, 4-17.

Rosenberg, S. M., Thulin, C. \& Harris, R. S. (1998). Transient and heritable mutators in adaptive evolution in the lab and in nature. Genetics 148, 1559-1566.

Samrakandi, M. M. \& Pasta, F. (2000). Hyperrecombination in Streptococcus pneumoniae depends on an atypical mutY homologue. J Bacteriol 182, 3353-3360.

Shaver, A. C., Dombrowski, P. G., Sweeney, J. Y., Treis, T., Zappala, R. M. \& Sniegowski, P. D. (2002). Fitness evolution and the rise of mutator alleles in experimental Escherichia coli populations. Genetics 162, 557-566.

Sniegowski, P. D., Gerrish, P. J. \& Lenski, R. E. (1997). Evolution of high mutation rates in experimental populations of E. coli. Nature 387, 703-705.

Sniegowski, P. D., Gerrish, P. J., Johnson, T. \& Shaver, A. (2000). The evolution of mutation rates: separating causes from consequences. Bioessays 22, 1057-1066. 
Tatusov, R. L., Fedorova, N. D., Jackson, J. D. \& 14 other authors (2003). The COG database: an updated version includes eukaryotes. BMC Bioinformatics 4, 41.

Thomas, S. R., Ray, A., Hodson, M. E. \& Pitt, T. L. (2000). Increased sputum amino acid concentrations and auxotrophy of Pseudomonas aeruginosa in severe cystic fibrosis lung disease. Thorax 55, 795-797.

Trong, H. N., Prunier, A. L. \& Leclercq, R. (2005). Hypermutable and fluoroquinolone-resistant clinical isolates of Staphylococcus aureus. Antimicrob Agents Chemother 49, 2098-2101.
Watson, M. E., Jr, Burns, J. L. \& Smith, A. L. (2004). Hypermutable Haemophilus influenzae with mutations in mutS are found in cystic fibrosis sputum. Microbiology 150, 2947-2958.

Young, D. M. \& Ornston, L. N. (2001). Functions of the mismatch repair gene mutS from Acinetobacter sp. strain ADP1. J Bacteriol 183, 6822-6831.

Zhao, J. \& Winkler, M. E. (2000). Reduction of GC $\rightarrow$ TA transversion mutation by overexpression of MutS in Escherichia coli K-12. J Bacteriol 182, 5025-5028. 